\title{
BMJ Open Impact of transitional care interventions on hospital readmissions in older medical patients: a systematic review
}

\author{
Lisa Fønss Rasmussen (10 , ${ }^{1,2}$ Louise Bang Grode, ${ }^{1}$ Jeppe Lange, ${ }^{2,3}$ Ishay Barat, ${ }^{1,2}$ \\ Merete Gregersen ${ }^{4}$
}

To cite: Fønss Rasmussen $L$, Grode LB, Lange J, et al. Impact of transitional care interventions on hospital readmissions in older medical patients: a systematic review. BMJ Open 2021;11:e040057. doi:10.1136/ bmjopen-2020-040057

- Prepublication history and additional materials for this paper is available online. To view these files, please visit the journal online (http://dx.doi. org/10.1136/bmjopen-2020040057).

Received 04 May 2020 Revised 10 December 2020 Accepted 16 December 2020

Check for updates

C Author(s) (or their employer(s)) 2021. Re-use permitted under CC BY-NC. No commercial re-use. See rights and permissions. Published by BMJ.

1Department of Research and Department of Medicine, Regional Hospital Horsens, Horsens, Denmark

${ }^{2}$ Department of Clinical

Medicine, Aarhus Universitet,

Aarhus, Denmark

${ }^{3}$ Department of Orthopedic

Surgery, Regional Hospital

Horsens, Horsens, Denmark

${ }^{4}$ Departments of Geriatrics,

Aarhus University Hospital,

Aarhus, Denmark

Correspondence to

Lisa Fønss Rasmussen;

lirasm@rm.dk

\section{ABSTRACT}

Objectives To identify and synthesise available evidence on the impact of transitional care interventions with both predischarge and postdischarge elements on readmission rates in older medical patients.

Design A systematic review.

Method Inclusion criteria were: medical patients $\geq 65$ years or mean age in study population of $\geq 75$ years; interventions were transitional care interventions between hospital and home with both predischarge and postdischarge components; outcome was hospital readmissions. Studies were excluded if they: included other patient groups than medical patients, included patients with only one diagnosis or patients with only psychiatric disorders. PubMed, The Cochrane Library, Embase, Cumulative Index to Nursing and Allied Health Literature (CINAHL) and Web of Science were searched from January 2008 to August 2019. Study selection at title level was undertaken by one author; the remaining selection process, data extraction and methodological quality assessment were undertaken by two authors independently. A narrative synthesis was performed, and effect sizes were estimated.

Result We identified 1951 records and included 11 studies: five randomised trials, four non-randomised controlled trials and two pre-post cohort studies. The 11 studies represent 15 different interventions and 29 outcome results measuring readmission rates within 7-182 days after discharge. Twenty-two of the 29 outcome results showed a drop in readmission rates in the intervention groups compared with the control groups. The most significant impact was seen when interventions were of high intensity, lasted at least 1 month and targeted patients at risk. The methodological quality of the included studies was generally poor.

Conclusion Transitional care interventions reduce readmission rates among older medical patients although the impact varies at different times of outcome assessment. High-quality studies examining the impact of interventions are needed, preferably complimented by a process evaluation to refine and improve future interventions.

PROSPERO registration number CRD42019121795.

\section{INTRODUCTION}

Transitional care interventions (TCIs) may be essential in older medical patients'

\section{Strengths and limitations of this study}

Focus on discharge interventions where intervention elements are provided both at the hospital and at home.

- High internal validity as Preferred Reporting Items for Systematic Reviews and Meta-Analyses guideline has been applied.

- Results may under-represent negative study findings as negative impacts often remain unpublished.

- Unplanned readmissions were the only outcome assessed in this review.

transition from hospital to home as they may prevent adverse events and unplanned hospital readmissions. These events can have detrimental consequences for the individual patient.

The global demography is radically changing. In the European Union, elderly above 65 years are estimated to account for $29.1 \%$ of the total population in 2080 compared with $19.2 \%$ in 2016. Additionally, the fraction of the population above 80 years is expected to double between 2016 and $2080 .{ }^{1}$ We may therefore expect a dramatic increase in healthcare service demands and costs. ${ }^{2}$ As these changes will bring substantial challenges to healthcare systems, ${ }^{34}$ the potential need for TCIs will also increase. Older people needing healthcare are often medical patients with several concurrent diseases, reduced physical or mental functionalities, limited ability to provide self-care and they are often living alone and need care from primary or secondary healthcare services. ${ }^{5}$

Older people with complex comorbid conditions are at high risk of adverse events and safety incidents immediately after their discharge from hospital. ${ }^{6}$

Unplanned readmission seems to be related to insufficient discharge planning, and unintended events during discharge and transition 
such as medication errors and inadequate communication between hospital and primary care professionals. ${ }^{7-10}$ By contrast, optimised, customised and patient-centred discharge planning and transitions may reduce length of hospital stay, risk of readmission, medication discrepancies and mortality; and may as well improve the patients' activity of daily living and reduce healthcare costs. ${ }^{11}$

One approach to addressing these challenges is to examine the impact of interventions aimed at reducing readmissions.

Previous systematic reviews have mainly evaluated the impact of hospital-based and/or home-based interventions on readmissions ${ }^{12} 13$ and included populations with specific conditions or both medical and surgical patients. To our knowledge, no systematic review has been conducted examining the impact of TCIs that take place in both hospital and home on older medical patients' readmission rates based on recent data.

The purpose of this systematic review is to evaluate the impact of TCIs with both predischarge and postdischarge elements on readmission among older medical patients.

\section{METHODS}

This review was registered in the PROSPERO database prior to data collection (CRD42019121795) ${ }^{14}$ It is presented in accordance with the Preferred Reporting Items for Systematic Reviews and Meta-Analyses (PRISMA). ${ }^{15}$

\section{Eligibility criteria}

We identified studies that aimed to reduce readmission rates through TCIs among older medical patients. The PICO process for framing the research question was applied ${ }^{16}$ and defined as:

1. Population: older medical patients discharged from a general medical ward or emergency department (ED).

2. Intervention: in the transitional phase between hospital and home which examined the impact of the intervention on readmission rates. The interventions had to include both predischarge and postdischarge components.

3. Comparison: usual care defined as standard care and treatment.

4. Outcome: unplanned readmission to hospital.

Selecting tool is displayed in online supplemental 1.

Studies were excluded if:

1. The population was aged under 65 years or the mean age was below 75 years.

2. They included other patient groups than medical patients (eg, surgical patients).

3. They included participants with only one medical diagnosis (International Classification of Disease-10th edition).

4. Studies included participants with psychiatric disorders only.

5. They compared interventions with anything other than usual care.
6. Readmission was not an outcome.

7. Intervention only included either predischarge or postdischarge components; they were reviews, case reports or case studies without comparison groups.

\section{Information sources}

To identify eligible studies, we searched the following bibliographic databases: PubMed, The Cochrane Library, Embase, CINAHL and Web of Science from January 2008 to August 2019. An extensive snowball search was performed where the reference lists from relevant studies, systematic reviews and included studies were examined. Additionally, publication lists from prominent researchers within the field were examined. Grey literature was searched in all relevant resources listed by Paez ${ }^{17}$ from January 2008 to August 2019. Authors from relevant study protocols and grey literature were contacted in order to examine whether the studies were published or study results were available. The Negative Results Scientific Journal was searched.

\section{Search strategy}

The search string (online supplemental 2) was developed in collaboration between the authors and a university research librarian. We used key terms, free textwords, subject headings, index terms and appertaining synonyms, which were identified through relevant theory and research. The searches were limited by only including studies published in English or Scandinavian languages. The bibliographic searches were conducted on 13 and 14 December 2018 and the searches were regularly updated until 31 August 2019.

\section{Study selection}

First, titles were screened for their potential relevance according to population and outcome by the first author (LFR). Second, two authors independently screened titles and abstracts for intervention eligibility (LFR and $M G)$. Third, an assessment of the full text was performed by two authors independently (always including LFR). In case of disagreement, a third author, who was chosen a priori, was consulted.

\section{Data extraction}

The Cochrane Data Extraction Form was modified to fit the present patient group and intervention type. ${ }^{18}$ Data from the included studies were extracted by two researchers independently (always including LFR). Extracted data included study characteristics and results such as author, year of publication, country, study design, setting, participants, study size, outcomes, follow-up time and impact of intervention in numbers and/or per cent. Only data on the outcome 'readmission' were extracted and analysed.

\section{Quality assessment}

'The Quality Assessment Tool for Quantitative Studies' (EPHPP) (online supplemental 3) was applied to 
assess bias in each study included in the review. The validated tool is recommended by The Cochrane Collaboration ${ }^{16} 19$ and provides a standardised means to assess study quality. The Effective Public Healthcare Panacea Project (EPHPP) assesses six methodological dimensions: selection bias, study design, confounders, blinding, data collection as well as withdrawals and dropouts. It distributes the overall methodological rating into a strong, moderate or weak measure of internal validity. ${ }^{20}$ Quality assessment will be conducted at study level. All studies meeting the inclusion criteria are included in the synthesis regardless of the results of the quality assessments. Quality assessment across studies will be analysed and the impact hereof will be discussed.

\section{Data synthesis}

Inspired by Pigott and Shepperd, ${ }^{21}$ the following aspects that can entail heterogeneity will be assessed: (1) context, (2) target population, (3) intervention, (4) methodological features and (5) researcher characteristics and reporting context. This assessment will result in a

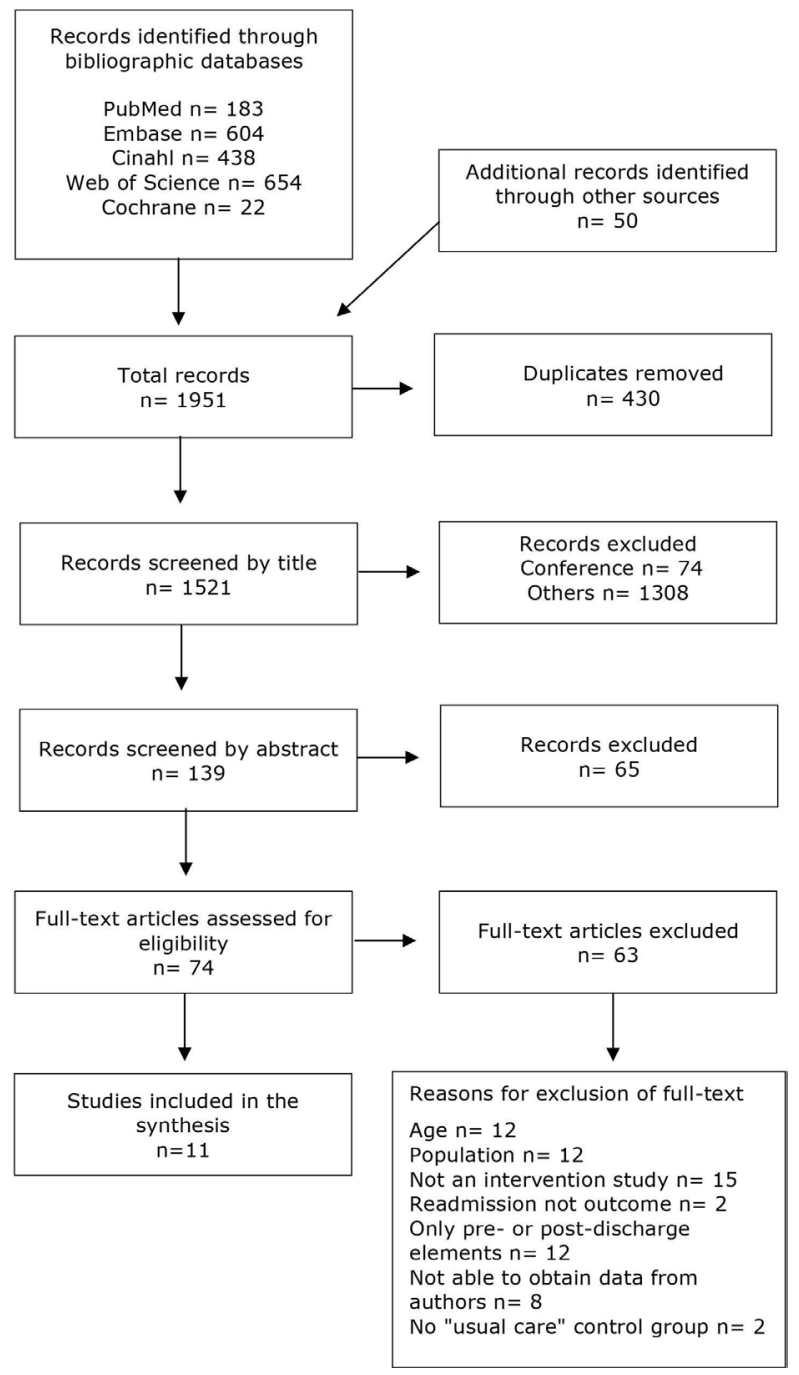

Figure 1 Flow chart of the study selection. descriptive synthesis or a meta-analysis of data. Risk estimates (RR) and their 95\% CIs are calculated (section 6.4.1 in Ref. 16) when possible and presented in forest plots stratified by subgroups. Only main results will be presented and discussed according to subgroups based on study-and intervention characteristics and review findings. The certainty of the synthesised results will be assessed using Grading of Recommendations Assessment, Development and Evaluation (GRADE) (section 14-2-1 in Ref. 16).

\section{Patient and public involvement}

Patients or the public were not involved in this study.

\section{RESULTS}

\section{Study selection}

In total, 1951 records were identified. Of those, 1901 records were identified through bibliographic databases and 50 records were found through other sources. After removing duplicates, 1521 records were screened by title and abstract. Seventy-four records were considered for full-text review of which 11 met the eligibility criteria (figure 1). The 63 remaining records were excluded due to participants' age, population, study design, readmission not listed as an outcome, only predischarge or postdischarge elements in the intervention, not able to obtain data from authors or no 'usual care' control group (online supplemental 4).

\section{Study characteristics}

The 11 included studies represented 15 different interventions and 29 outcome assessments measuring readmission rates at different time points. Two studies were multiarm studies. ${ }^{22}{ }^{23}$ Study characteristics are shown in table 1 . Five of the included studies were randomised trials (RCTs), ${ }^{23-27}$ four non-randomised controlled trials (NRCTs) ${ }^{22} 28-30$ and two pre-post cohort studies. ${ }^{31} 32$

Sample sizes of individual studies ranged from 41 to 19157 . Allocation to intervention or control group was performed on an individual level. The majority of studies reported that readmissions were unplanned or acute. ${ }^{23-28} 32$

Outcome assessments were conducted 7-182 days after discharge. In addition to readmission, the 11 studies assessed outcomes according to physical functioning ${ }^{24}{ }^{27}$; cognitive functioning ${ }^{24}$; quality of life ${ }^{222527}$; time to either hospital readmission or discharge from nursing homes ${ }^{2428}$; self-efficacy ${ }^{22}$; self-rated health ${ }^{22}$; visits to EDs, general

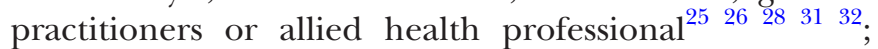
length of stay ${ }^{27}{ }^{31}$; comorbidity ${ }^{27}$; cost-effectiveness ${ }^{27}$ and mortality. ${ }^{24}{ }^{28}$ Three studies took place in USA, ${ }^{26} 2930$ two in Australia, ${ }^{23}{ }^{25}$ two in Hong Kong, ${ }^{22} 31$ one in the Netherlands, ${ }^{24}$ one in the UK, ${ }^{27}$ one in Denmark ${ }^{28}$ and one in New Zealand. ${ }^{32}$ The studies were published between 2009 and 2018. 


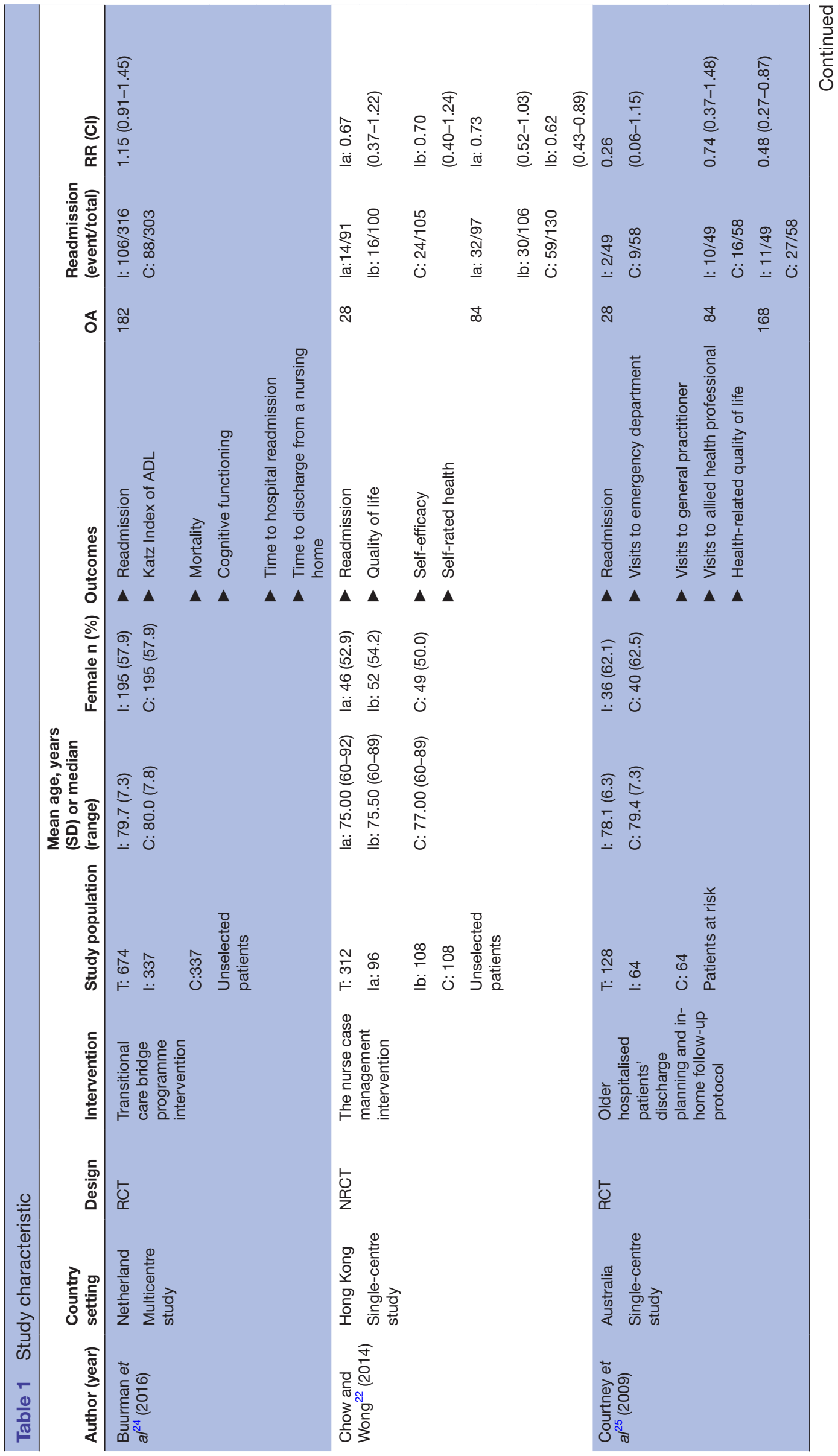




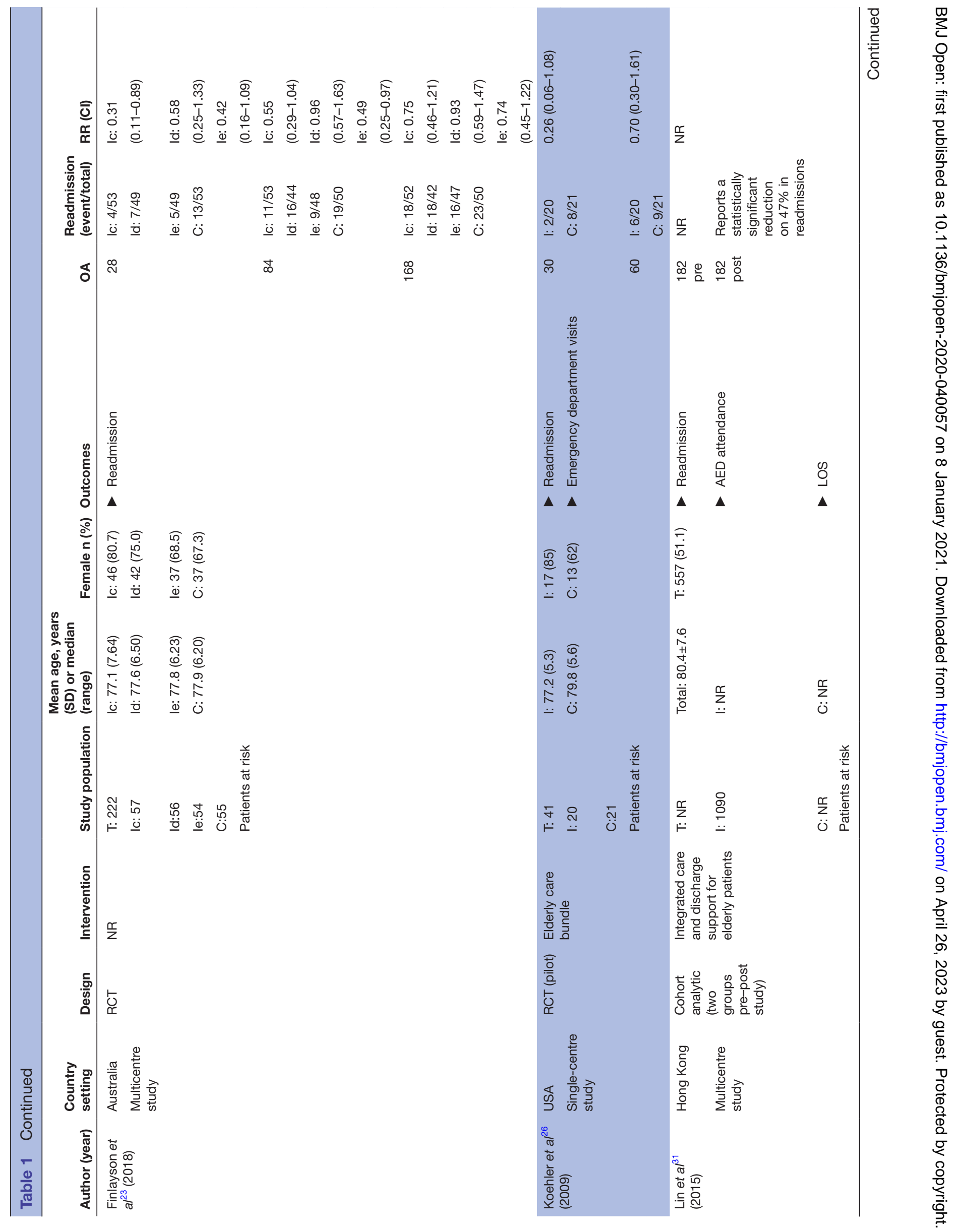




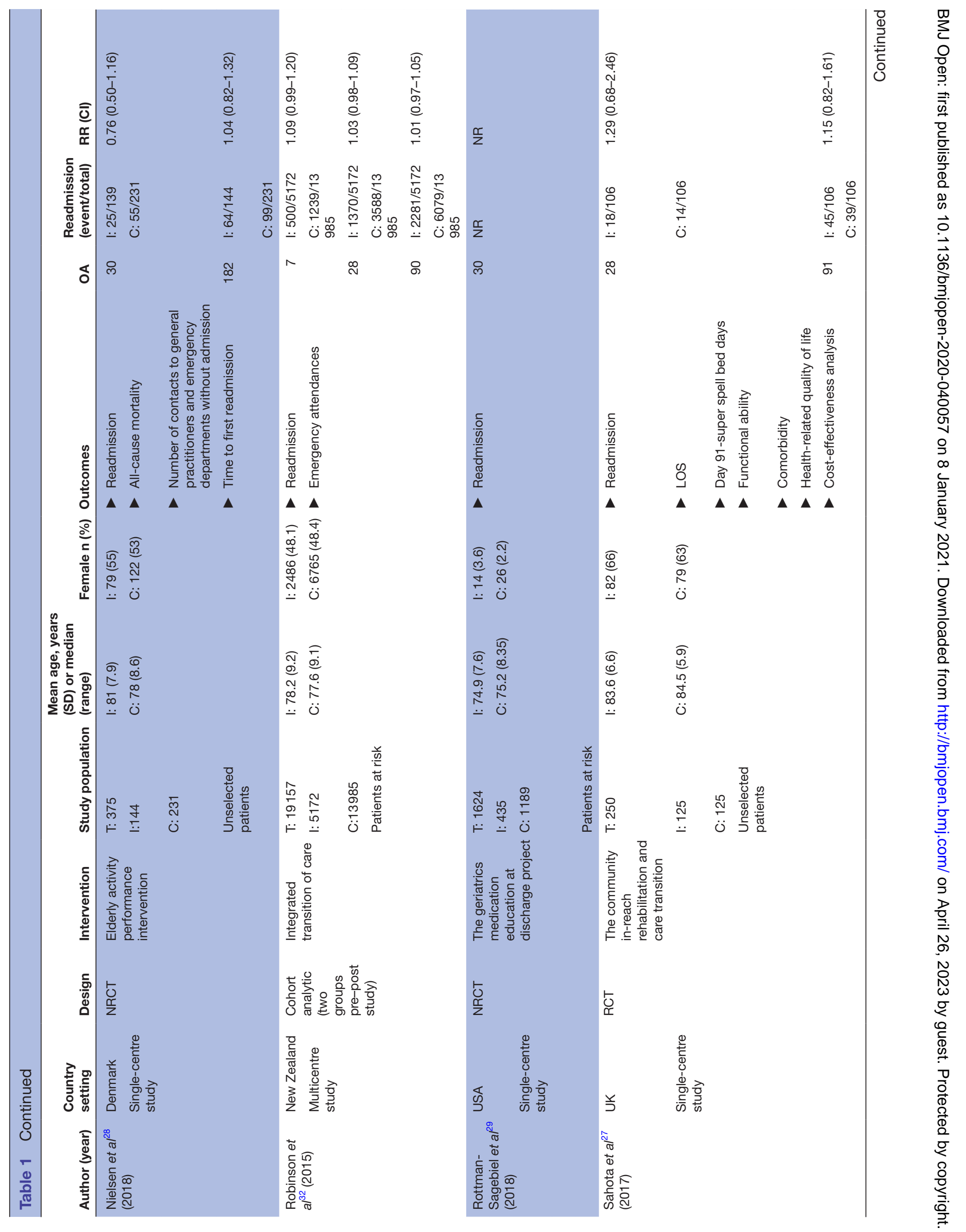




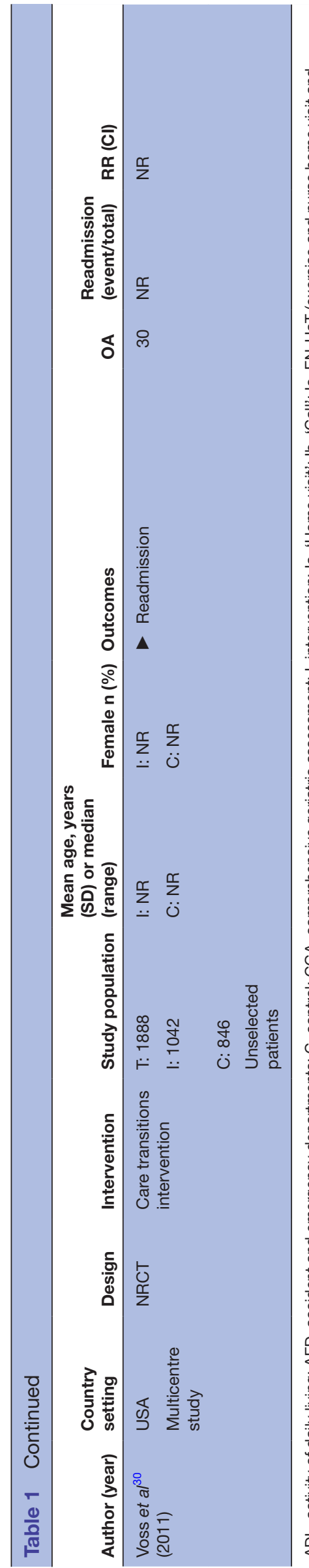

\section{Study population}

In total, approximately 24500 patients were evaluated in our review; 8800 in the intervention groups and 15700 in the control groups. The mean age of the participants in the intervention groups was approximately 78 years (range 74.9-83.6), while the mean age among the control groups was approximately 79 years (range 75.2-84.5). Approximately 59\% (range 3.6-85) of the participants in the intervention groups were women and 52\% (range 2.2-67) of the participants in the control group were women.

\section{TCls}

All studies included predischarge and postdischarge components and some included bridging components. The most pronounced difference between the interventions was in the predischarge phase, whereas elements in the bridging and postdischarge phase were somewhat similar. Table 2 outlines the intervention components, and online supplemental 5 contains detailed descriptions of the interventions.

\section{Predischarge components}

Across studies, predischarge components consisted of health record or plan, discharge planning, involvement of caregivers, concerns or barriers, patient education, physical exercise (physical exercise (muscle strength, stretch, balance and walking), medication reconciliation, counselling, nutritional screening, predischarge home visit or patient assessments (comprehensive geriatric assessment, problem classification scheme, assessment of patients' performance of daily activities and assessment of motor and process skills).

\section{Bridging components}

Bridging components consisted of written handover between hospital and primary sector, telephone handover or in-person handover.

\section{Postdischarge components}

Postdischarge components comprised of home visits, telephone follow-up, referral to additional healthcare services (rehabilitation, early specialist follow-up, general practitioner or other community services), nurse availability from hospital discharge until 7 days after discharge or rapid and intensive community support.

\section{Synthesis of results}

All five aspects that may introduce heterogeneity were analysed and a pronounced diversity between the included studies was found. Additionally, some studies had incomplete reporting of study effects. Therefore, because of the heterogeneity, a meta-analysis could not be conducted.

Considering the impact on outcome level, 22 of the 29 outcome results $(76 \%)$ showed a positive impact on readmission in the intervention groups compared with the control groups. Three studies did not report readmission rates but reported a positive impact on readmission rates, 


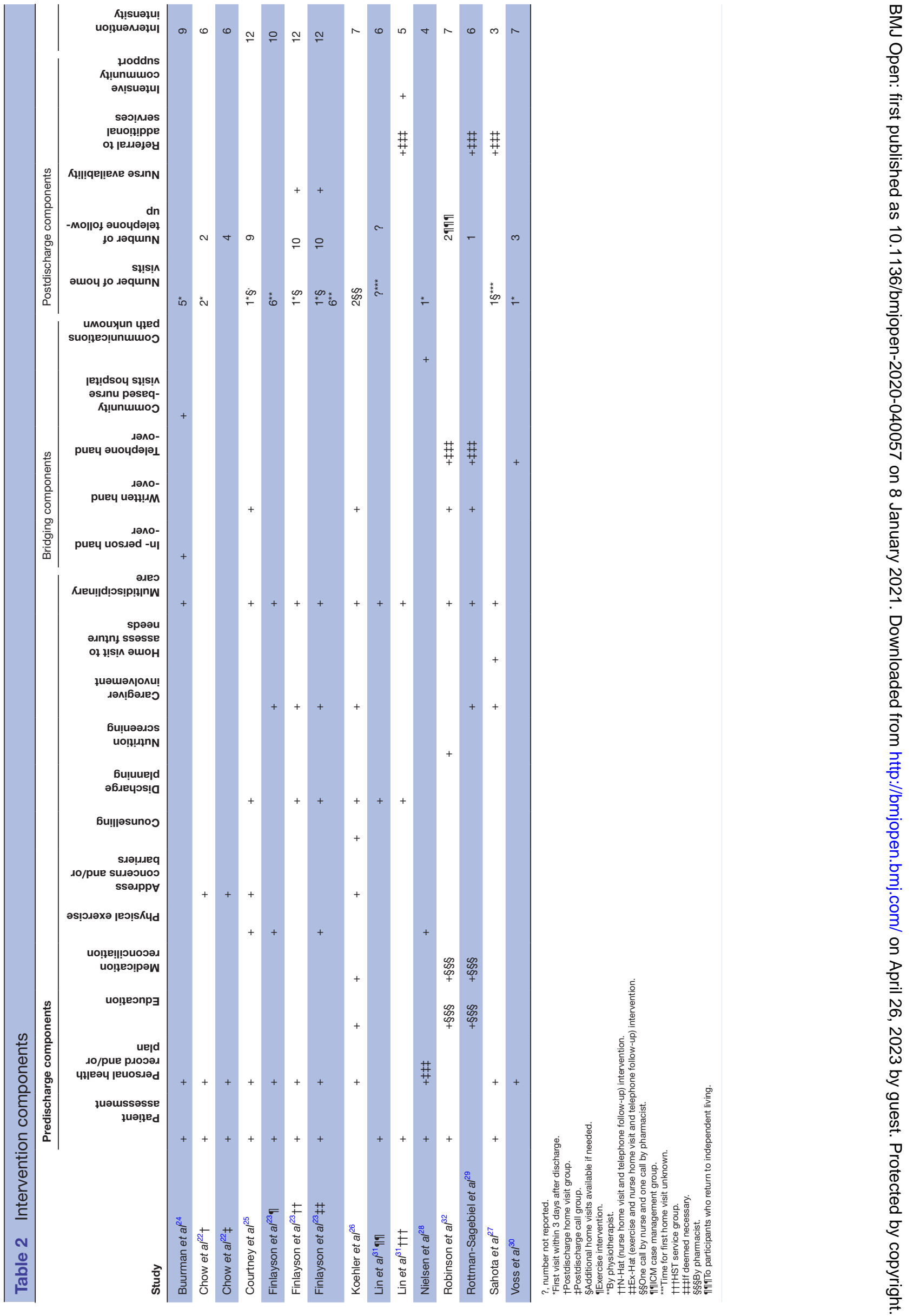


and it was not possible to obtain these numbers from the authors. ${ }^{29-31}$ It was not possible to calculate RRs and their 95\% CIs for those studies, and they are therefore not presented in the forest plots.

The impact size (RR) from individual studies was calculated using the reported readmission rates from eight studies. RRs ranged from 0.26 to 1.29 . Nineteen outcome results were $<1$ of which one was close to $1 \quad(R R=0.96)$. Seven estimates were $>1$ of which three were close to 1 $(\mathrm{RR}=1.01,1.03,1.04)$. Of the 29 outcome results, five were statistically significant. ${ }^{22} 23252631$

Considering the impact on study level, seven of the 11 studies showed an entirely positive impact on readmissions, ${ }^{22} 232526$ 29-31 while one study presented both a positive impact and no impact, ${ }^{28}$ and three studies showed no impact at all. ${ }^{24} 27$

\section{Subgroup analysis}

Study population

Online supplemental 6 exhibits impact according to the included study population. There is a clear difference in impacts between the groups. In total, 14 of 17 (82\%) interventions including 'patients at risk' reported positive impacts on readmission rates. In contrast, only five out of nine $(56 \%)$ interventions including 'unselected patients' reported positive impacts. In addition, the positive impacts were larger among interventions including patients at risk than among interventions with unselected patients. Three of the four statistically significant results are found among the patients at risk group. However, the 95\% CIs are wider among patients at risk compared with the unselected patients indicating less precise effect estimates.

\section{Intervention intensity}

Calculation of intervention intensity was inspired by Verhaegh et al. ${ }^{33}$ Ten interventions were categorised as low intensity ${ }^{22} 26-32$ and five were categorised as high intensity. ${ }^{23-25}$

Online supplemental 7 illustrates a clear difference. The vast majority of interventions with a high intensity reported a positive impact on readmission rates whereas only half of the interventions with a low intensity showed a positive impact. The impacts were larger and statistically significant among high-intensive interventions.

\section{Length of support}

The interventions lasted from enrolment until 1 day to 6 months after hospital discharge. Four studies lasted between 1 and 7 days, ${ }^{28} 2932$ two studies lasted 28 and 30 days, ${ }^{30}$ one study 84 days, ${ }^{31}$ two 168 days, ${ }^{24}$ one 182 days $^{25}$ and one study did not report the duration of the intervention. ${ }^{27}$ Online supplemental 8 displays the impact on readmission rates according to the duration of the interventions. A short length of support is associated with less or no impact on readmission rates. A length of support of 1 month or more is associated with positive, larger and statistically significant impacts.

\section{Country of origin}

Studies conducted outside the European countries seemed to have a greater impact on readmissions than studies conducted within the European Union and all statistically significant results are found in studies conducted in non-European countries (online supplemental 9).

\section{Outcome assessment}

The impact on readmissions was largest within 30 days after hospital discharge. The impact decreased hereafter and was similar between 1 month and 6 months after discharge. Most statistically significant results are found when outcomes are assessed between 1 and 3 months (online supplemental 10).

\section{Quality assessment within studies}

Of the 11 studies, two studies were assessed to have a strong methodological quality, ${ }^{22} 25$ three had a moderate 242732 and six had a weak methodological quality. ${ }^{232628-31}$ Of the RCTs, one had a strong, ${ }^{25}$ two had a moderate ${ }^{24} 27$ and two had a weak quality. ${ }^{23}{ }^{26}$ Of the NRCTs, one study had a strong ${ }^{22}$ and three had a weak quality. ${ }^{28-30}$ One of the pre-post cohort studies had a moderate ${ }^{32}$ and one had a weak quality $^{31}$ (table 3 ).

\section{Quality assessment across studies}

The majority of the studies did not meet the criteria in the components selection bias and blinding and were thus rated moderate or weak. The vast majority of studies met the criteria of study design, confounders and data collection and were therefore rated strong (figure 2).

\section{DISCUSSION}

We found that the majority of interventions in the transitional phase between hospital and home appears to reduce readmission rates among older patients discharged from a medical ward.

However, some studies reported both a positive impact and no impact on the readmission rate following similar care interventions. These divergent results may have several plausible explanations as discussed below.

\section{Explanations related to study characteristics \\ Country of origin}

Studies conducted in European countries have less impact than studies conducted in non-European countries. The impact of complex interventions is, among others, altered by the context of the implementation, ${ }^{3435}$ and differences in impact between countries may therefore be explained by diversity in the social, political, economic, clinical and geographical setting. The accessibility, type, character, quality and overall comprehensiveness of healthcare services provided may also play a role. ${ }^{36}$ The USA and Australia have a long history of discharge planning and transitional care, and these countries therefore have a high quantity of research as well as refined strategies and guidelines. $^{37-39}$ 
Table 3 Quality assessment

\begin{tabular}{|c|c|c|c|c|c|c|c|}
\hline Author & $\begin{array}{l}\text { Selection } \\
\text { bias }\end{array}$ & $\begin{array}{l}\text { Study } \\
\text { design }\end{array}$ & Confounders & Blinding & $\begin{array}{l}\text { Data collection } \\
\text { methods }\end{array}$ & $\begin{array}{l}\text { Withdrawals } \\
\text { and drop-outs }\end{array}$ & $\begin{array}{l}\text { Global } \\
\text { rating }\end{array}$ \\
\hline Buurman et $a l^{24}$ & Moderate & Strong & Strong & Strong & Strong & Weak & Moderate \\
\hline Courtney et $a l^{25}$ & Moderate & Strong & Strong & Moderate & Strong & Moderate & Strong \\
\hline Finlayson et $a l^{23}$ & Weak & Strong & Strong & Weak & Weak & Moderate & Weak \\
\hline Nielsen et $\left.a\right|^{28}$ & Strong & Strong & Strong & Weak & Strong & Weak & Weak \\
\hline Robinson et a/ ${ }^{32}$ & Strong & Moderate & Strong & Weak & Strong & Strong & Moderate \\
\hline $\begin{array}{l}\text { Rottman-Sagebiel } \\
\text { et } a l^{29}\end{array}$ & Weak & Strong & Strong & Weak & Weak & Weak & Weak \\
\hline
\end{tabular}

\section{Study population}

The impact on readmissions is greater among patients at risk than among 'unselected older patients'. This is to be expected as readmission rates are higher among patients at risk than among unselected patients. Furthermore, patients at risk are more frail and may have a higher degree of morbidity, which could have affected the risk of readmission. ${ }^{40}$

\section{Readmission rates prior to the study}

Preintervention readmission rates may mirror differences in impact between studies. It may be assumed that hospitals with low preintervention readmission rates may experience no reduction of postintervention readmission rates as the remaining readmissions may not be preventable. In contrast, it may also be assumed that hospitals with high preintervention readmission rates will achieve a reduction in postintervention readmission rates.

\section{Sample size}

Several studies had small sample sizes. As seen in table 1, studies with small sample sizes reported a stronger impact than studies with larger sample sizes. This is in line with Dechartres et al who also reported larger effect sizes in small-to-moderate-sized trials than in larger trials. ${ }^{41}$ One

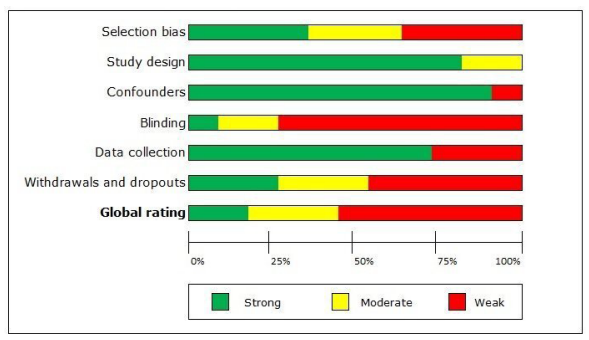

Figure 2 Quality assessment across studies. The green colour indicates strong methodological quality, yellow indicates moderate quality and red indicates weak quality across studies. reason may be that small studies are more prone to publication bias than larger studies. ${ }^{42}$

\section{Explanations related to review findings}

Interventions

Our findings suggest that the intensity of interventions influences the impact on readmission rates as highintensity interventions generally have a stronger impact than low-intensity interventions. This is in line with previous findings presented by Verhaegh et $a l^{33}$ The higher quantity of elements means that more aspects in a complex patient cohort and complex settings can be addressed.

Intervention components that at first glance appear to be similar across studies may comprise different features. The diverging impact on readmissions may be affected by differences in intervention contents across studies. It is not possible to make an intervention component analysis across studies that evaluates which components positively affect readmissions. However, some trends are seen in table 2. Interventions with a positive impact on readmission comprise the following components: patient assessment, personal health record or plan, concerns and barriers, discharge planning, caregiver involvement, home visits and telephone follow-up.

\section{Intervention fidelity}

The impact on readmission rates may be affected by the fidelity of the interventions. ${ }^{43}$ However, several studies do not describe intervention fidelity, which may be due to inconsistency in monitoring of the implementation or lack of transparency in reporting the study. These shortcomings thus hinder assessment of whether lack of positive impact is caused by poor implementation of the intervention or if the intervention did not work.

\section{Outcome assessment}

The timing of the outcome assessment has an important bearing on the possible preventive readmission rate. ${ }^{44}$ 
The findings of this review suggest that interventions have the largest impact within the first 30 days after discharge. A potential impact is likely to have obliterated if outcome is assessed later than 30 days after discharge.

\section{Residual confounding}

Several of the included studies did not adjust for potential confounders such as length of stay, fall within the past 12 months, living conditions, prior admissions, poor overall health condition and functional disability. ${ }^{45-47}$ This could have affected the internal validity and the impact on readmission rates. The lack of analysis adjusting for potential confounders is highlighted in previous studies, ${ }^{48}$ underlining the problematic trend in this research area.

\section{Risk of bias}

Differences in the quality assessment and thus risk of bias may explain the variation in the impact of the included studies. ${ }^{16}$ Online supplemental 11 shows that studies assessed to have weak and strong methodological quality have a positive impact on readmission rates whereas studies with moderate quality have no impact.

\section{Statistical analysis}

Several authors fail to report whether the statistical analysis is performed based on the first readmission. One patient may therefore represent several readmissions within a specific follow-up period. If this is the case, results from those studies may report a higher readmission rate and thus greater impact than studies using unique observations in their statistical analysis.

\section{Quality assessment}

The methodological quality of the majority of the included studies was low, thus indicating a high risk of bias. Low global rating can reflect both the methodological quality and insufficient reporting of the methodology. The latter makes it difficult to accurately assess the true quality and thus the risk of bias. If the low rating is caused by insufficient methodological reporting, it may have no effect on the impact. However, if the low rating reflects methodological problems, it probably underestimates the true impact of the interventions.

The component 'blinding' represents the weakest rating. Blinding of participants was not always possible due to the nature of the interventions. Blinding is crucial in pharmaceutical trials and in other clinical studies. However, little is known about the benefits or disadvantages of blinding participants in complex interventions and how bias due to blinding may affect the results. Blinding outcome assessors may not be crucial when outcome is collected through digital records.

\section{COMPARISON WITH PREVIOUS RESEARCH}

Prior systematic reviews included other study populations such as surgical patients or patients with only one medical condition. The present review includes a broader population, namely older medical patients. Direct comparison of the present findings with previous findings is therefore difficult. The present review suggests that TCIs reduce the risk of readmission among older medical patients.

These findings are in line with findings in similar reviews that found positive effects of TCIs on hospital readmission. ${ }^{49-51}$ These reviews also found that the included studies had a very-low-to-moderate methodological quality.

\section{LIMITATION OF INCLUDED EVIDENCE BASE}

Transparency across studies is lacking even if the Consolidated Standards of Reporting Trials statement recommends reporting a sufficient description of interventions. ${ }^{52} \mathrm{~A}$ call for more transparency in clinical trials and adherence to appropriate guidelines is also reported elsewhere. ${ }^{53}$ If the template for the intervention description and replication is followed, the replicability may improve, thus making it possible to build on prior research findings. ${ }^{54}$

Evaluating TCIs provides insight into whether interventions reduce readmissions among intervention groups compared with control groups. However, we gain no knowledge about causality between exposure and outcome. In complex interventions, such as TCIs, it is difficult to evaluate which mechanisms or components result in change. ${ }^{55}$ A process evaluation may have captured the fidelity of the interventions and may thus have provided insight into which mechanisms and components actually work. ${ }^{56}$ Process evaluation has previously been requested, ${ }^{57}$ stressing the unmet need to identify essential components in TCIs. We have requested missing intervention details and other relevant data from authors; some requests were met while others were not. Lastly, all GRADE domains except one (indirectness of evidence) were assessed and found to downgrade the evidence and hence, lower the certainty of the evidence of this review.

\section{Strengths and limitations of this review}

The study has several strengths. This review adhered to PRISMA $^{15}$ and synthesis without meta-analysis ${ }^{58}$ which ensures that all important methodological considerations were made. By reducing the risk of bias in this review, systematic errors were minimised during all stages of the process.

Despite these strengths, we note some limitations. The eligibility criteria were somewhat narrow, which limited the number of included studies. This, however, was deemed necessary to meet the need for evidence focusing on clinical settings where a large number of hospital wards are general medical wards.

Only studies published in English, Danish, Norwegian or Swedish were eligible for inclusion in this review. Therefore, studies published in other languages were omitted, potentially excluding useful evidence.

This review only focuses on hospital readmission as an outcome although the included studies evaluated the intervention impact on multiple outcomes. It is therefore 
possible that some studies report a positive impact in terms of other outcomes than readmission.

Positive findings are more likely to be published in English language journals, whereas negative findings are more often published in local-language journals. ${ }^{59}$ Publication bias is therefore likely present. During this review process, we have contacted several researchers who had registered TCIs on ClinicalTrials.gov. All researchers who found no or negative impact either struggled to publish or decided not to publish. The above-mentioned issues may result in an underrepresentation of negative results in the evidence base and thus in this present review.

\section{Implications and future research}

Future research projects may benefit from the knowledge gained from the present review when designing and developing new studies. Knowing that interventions with a minimum duration of 1 month that have a high intensity and target high-risk patients may result in more effective interventions.

This review highlights an unmet need for studies of high methodological quality that evaluate the impact of TCIs among older medical patients. Therefore, future research can benefit from a higher level of adherence to relevant guidelines and more detailed descriptions of (1) interventions and comparison groups, (2) the implementation process and (3) actions taken to minimise bias and confounding. Further research should be undertaken to investigate the process evaluations of complex interventions to identify how and why interventions either work or do not work. To develop a broader picture of TCIs, additional studies need to focus on psychological outcomes. The societal cost of such interventions versus individual and societal benefits needs to be further evaluated. Highrisk patients such as physically disabled, chronically ill patients may benefit from these kinds of interventions regardless of age and comorbidity. ${ }^{60}$

\section{CONCLUSION}

The majority of TCIs have a positive impact on readmission rates among older medical patients, although the most significant impact was seen within 30 days after hospital discharge. Therefore, we believe that the current evidence supports recommending transitional care that includes both predischarge and postdischarge components. However, no evidence for recommending a specific intervention exists. The key finding shows an apparent pronounced positive impact among patients categorised as patients at risk in 'high-intensity' interventions and in interventions with duration of 1 month or more. This, however, should be seen in the light of the fact that only 11 studies met the inclusion criteria and a low certainty of evidence according to the GRADE approach.

Contributors All authors listed have contributed sufficiently to the project to be included as authors according to the Vancouver Guideline. All authors have participated in the development of the study, writing the draft and all have approved the final version. LFR: first author writing the manuscript, screening records for inclusion, data extraction of included studies, quality assessment of included studies. LBG, JL, IB, MG: initial development of the study, screening records for inclusion, data extraction of included studies, quality assessment of included studies, reading and giving feedback on the manuscript and approving the final manuscript.

Funding This work was supported by the 'Fund for advancement of health research in Central Denmark Region'. Grant number N/A.

Competing interests None declared.

Patient consent for publication Not required.

Provenance and peer review Not commissioned; externally peer reviewed

Data availability statement All data relevant to the study are included in the article or uploaded as supplementary information.

Supplemental material This content has been supplied by the author(s). It has not been vetted by BMJ Publishing Group Limited (BMJ) and may not have been peer-reviewed. Any opinions or recommendations discussed are solely those of the author(s) and are not endorsed by BMJ. BMJ disclaims all liability and responsibility arising from any reliance placed on the content. Where the content includes any translated material, BMJ does not warrant the accuracy and reliability of the translations (including but not limited to local regulations, clinical guidelines, terminology, drug names and drug dosages), and is not responsible for any error and/or omissions arising from translation and adaptation or otherwise.

Open access This is an open access article distributed in accordance with the Creative Commons Attribution Non Commercial (CC BY-NC 4.0) license, which permits others to distribute, remix, adapt, build upon this work non-commercially, and license their derivative works on different terms, provided the original work is properly cited, appropriate credit is given, any changes made indicated, and the use is non-commercial. See: http://creativecommons.org/licenses/by-nc/4.0/.

ORCID iD

Lisa Fønss Rasmussen http://orcid.org/0000-0001-9405-9158

\section{REFERENCES}

1 World Health Organisation. World report on ageing and health, 2015.

2 Lehnert T, Heider D, Leicht $\mathrm{H}$, et al. Review: health care utilization and costs of elderly persons with multiple chronic conditions. Med Care Res Rev 2011;68:387-420.

3 Holm EA, Rønholt F, eds. Geriatri (Geriatrics), 2016.

4 Sundhedsdatastyrelsen. AEldre medicinske patienters kontakt med det regionale sundhedsvæsenog den kommunale pleje (older medical patients' contact to the regional healthcare system and the home care): Afdelingen for Sundhedsanalyser og lægemiddelstatistik, 2016.

5 Sundheds- og ÆEldreministeriet. Styrket indsats for den ældre medicinske patient. National handlingsplan 2016 (Improved effort for the elderly medical patient. National action plan), 2016.

6 Coleman EA, Min S-joon, Chomiak A, et al. Posthospital care transitions: patterns, complications, and risk identification. Health Serv Res 2004;39:1449-66.

7 Jencks SF, Williams MV, Coleman EA. Rehospitalizations among patients in the medicare fee-for-service program. $N$ Engl J Med 2009;360:1418-28.

8 Mesteig M, Helbostad JL, Sletvold O, et al. Unwanted incidents during transition of geriatric patients from hospital to home: a prospective observational study. BMC Health Serv Res 2010;10.

9 National Transitions of Care Coalition. Improving transitions of care. Findings and considerations of the 'vision of the National of care coalition', 2010.

10 Russell L, Doggett J, Dawda P. Patient safety - handover of care between primary and acute care. policy review and analysis. Prepared for the National lead clinicians group by the Australian primary health care research Institute, 2013.

11 Saleh SS, Freire C, Morris-Dickinson G, et al. An effectiveness and cost-benefit analysis of a hospital-based discharge transition program for elderly Medicare recipients. J Am Geriatr Soc 2012;60:1051-6.

12 Fan L, Lukin W, Zhao J, et al. Interventions targeting the elderly population to reduce emergency department utilisation: a literature review. Emerg Med J 2015;32:738-43.

13 Linertová R, García-Pérez L, Vázquez-Díaz JR, et al. Interventions to reduce hospital readmissions in the elderly: in-hospital or home care. A systematic review. J Eval Clin Pract 2011;17:1167-75.

14 National Institute for Health Research. PRPSPERO International prospective register of systematic reviews. Available: https://www. crd.york.ac.uk/prospero/ 
15 Moher D, Liberati A, Tetzlaff J, et al. Preferred reporting items for systematic reviews and meta-analyses: the PRISMA statement. $B M J$ 2009;339:b2535.

16 Higgins JPT, Thomas J, Chandler J. Cochrane handbook for systematic reviews of interventions version 6.0 (updated July 2019): cochrane, 2019.

17 Paez A. Gray literature: an important resource in systematic reviews. J Evid Based Med 2017;10:233-40.

18 Cochrane Effective Practice and Organisation of Care (EPOC). Data collaction form.: EPOC resources for review authors, 2017. Available: https://epoc.cochrane.org/resources/epoc-resources-reviewauthorsMarch

19 Armstrong R, Waters E, Jackson N. Guidelines for systematic reviews of health promotion and public health interventions. version 2. Australia: Melbourne University, 2007.

20 Deeks J, Dinnes J, D'Amico R. Evaluating non-randomised intervention studies. 7. Health Technol Assess England, 2003.

21 Pigott T, Shepperd S. Identifying, documenting, and examining heterogeneity in systematic reviews of complex interventions. J Clin Epidemiol 2013;66:1244-50.

22 Chow SKY, Wong FKY. A randomized controlled trial of a nurse-led case management programme for hospital-discharged older adults with co-morbidities. J Adv Nurs 2014;70:2257-71.

23 Finlayson K, Chang AM, Courtney MD, et al. Transitional care interventions reduce unplanned Hospital readmissions in high-risk older adults. BMC Health Serv Res 2018;18:956.

24 Buurman BM, Parlevliet JL, Allore HG. Comprehensive geriatric assessment and transitional care in acutely hospitalized patients the transitional care bridge randomized clinical trial. JAMA Intern Med 2016;176:302-9.

25 Courtney M, Edwards $\mathrm{H}$, Chang $\mathrm{A}$, et al. Fewer emergency readmissions and better quality of life for older adults at risk of hospital readmission: a randomized controlled trial to determine the effectiveness of a 24-week exercise and telephone follow-up program. J Am Geriatr Soc 2009;57:395-402.

26 Koehler BE, Richter KM, Youngblood L, et al. Reduction of 30-day postdischarge hospital readmission or emergency department (ED) visit rates in high-risk elderly medical patients through delivery of a targeted care bundle. J Hosp Med 2009;4:211-8.

27 Sahota O, Pulikottil-Jacob R, Marshall F. The community inreach rehabilitation and care transition (CIRACT) clinical and cost-effectiveness randomisation controlled trial in older people admitted to hospital as an acute medical emergency. Age Ageing 2017;46:26-32.

28 Nielsen L, Maribo T, Kirkegaard H, et al. Effectiveness of the elderly activity performance intervention on elderly patients discharge from a short-stay unit at the emergency department: a quasi-experimental trial. Clin Interv Aging 2018;13:737-47.

29 Rottman-Sagebiel R, Cupples N, Wang CP. A pharmacist-led transitional care program to reduce hospital readmissions in older adults. Fed Pract 2018;35:42-50.

30 Voss R, Gardner R, Baier R. The care transitions intervention: translating from efficacy to effectiveness. Arch Intern Med 2011;171:1232-7.

31 Lin FO, Luk JK, Chan TC. Effectiveness of a discharge planning and community support programme in preventing readmission of highrisk older patients. Hong Kong Med J 2015;21:208-16.

32 Robinson TE, Zhou L, Kerse N, et al. Evaluation of a new Zealand program to improve transition of care for older high risk adults. Australas J Ageing 2015;34:269-74

33 Verhaegh KJ, MacNeil-Vroomen JL, Eslami S, et al. Transitional care interventions prevent hospital readmissions for adults with chronic illnesses. Health Aff 2014:33:1531-9.

34 Pfadenhauer LM, Gerhardus A, Mozygemba K, et al. Making sense of complexity in context and implementation: the context and implementation of complex interventions $(\mathrm{ClCl})$ framework. Implementation Sci 2017;12:21.

35 Wells M, Williams B, Treweek S, et al. Intervention description is not enough: evidence from an in-depth multiple case study on the untold role and impact of context in randomised controlled trials of seven complex interventions. Trials 2012;13:95.

36 Krumholz HM, Wang K, Lin Z, et al. Hospital-readmission risk - isolating hospital effects from patient effects. N Engl J Med 2017;377:1055-64.
37 Bixby MB, Naylor MD. The transitional care model (TCM): hospital discharge screening criteria for high risk older adults. Medsurg Nurs 2010;19:62-3.

38 Naylor M, Brooten D, Jones R. Comprehensive discharge planning for the hospitalized elderly. A randomized clinical trial. Ann Intern Med 1994;120:999-1006.

39 Parry C, Coleman EA, Smith JD, et al. The care transitions intervention: a patient-centered approach to ensuring effective transfers between sites of geriatric care. Home Health Care Serv Q 2003;22:1-17.

40 Stillman GR, Stillman AN, Beecher MS. Frailty is associated with early Hospital readmission in older medical patients. J Appl Gerontol 2019:733464819894926

41 Dechartres A, Trinquart L, Boutron I, et al. Influence of trial sample size on treatment effect estimates: meta-epidemiological study. BMJ 2013;346:f2304.

42 Slavin R, Smith D. The relationship between sample sizes and effect sizes in systematic reviews in education. Educ Eval Policy Anal 2009;31:500-6.

43 Horner S, Rew L, Torres R. Enhancing intervention fidelity: a means of strengthening study impact. J Spec Pediatr Nurs 2006;11:80-9.

44 Fahlberg B. Preventing readmissions through transitional care. Nursing 2017;47:12-14.

45 Hallgren J, Aslan AKD. Risk factors for hospital readmission among Swedish older adults. Eur Geriatr Med 2018;9:603-11.

46 Hughes LD, Witham MD. Causes and correlates of 30 day and 180 day readmission following discharge from a medicine for the elderly rehabilitation unit. BMC Geriatr 2018;18:197.

47 Pedersen MK, Meyer G, Uhrenfeldt L. Risk factors for acute care hospital readmission in older persons in Western countries: a systematic review. JBI Database System Rev Implement Rep 2017;15:454-85.

48 Hughes JM, Freiermuth CE, Shepherd-Banigan M, et al. Emergency department interventions for older adults: a systematic review. J Am Geriatr Soc 2019;67:1516-25.

49 Le Berre M, Maimon G, Sourial N, et al. Impact of transitional care services for chronically ill older patients: a systematic evidence review. J Am Geriatr Soc 2017;65:1597-608.

50 Mabire C, Dwyer A, Garnier A, et al. Effectiveness of nursing discharge planning interventions on health-related outcomes in discharged elderly inpatients: a systematic review. JBI Database System Rev Implement Rep 2016;14:217-60.

51 Weeks LE, Macdonald M, Martin-Misener R, et al. The impact of transitional care programs on health services utilization in community-dwelling older adults: a systematic review. JBI Database System Rev Implement Rep 2018;16:345-84.

52 Schulz KF, Altman DG, Moher D. CONSORT 2010 statement: updated guidelines for reporting parallel group randomised trials. BMC Med 2010;8:18.

53 Ramírez JH. Lack of transparency in clinical trials: a call for action. Colomb Med 2013:44:243-6.

54 Hoffmann TC, Glasziou PP, Boutron I, et al. Better reporting of interventions: template for intervention description and replication (TIDieR) checklist and guide. BMJ 2014;348:g1687.

55 Craig P, Dieppe P, Macintyre S, et al. Developing and evaluating complex interventions: the new medical research council guidance. BMJ 2008;337:a1655

56 Moore GF, Audrey S, Barker M, et al. Process evaluation of complex interventions: medical research council guidance. BMJ 2015;350:h1258.

57 Mansah M, Fernandez R, Griffiths R, et al. Effectiveness of strategies to promote safe transition of elderly people across care settings. JBI Libr Syst Rev 2009;7:1036-90.

58 Campbell M, McKenzie JE, Sowden A, et al. Synthesis without metaanalysis (SWiM) in systematic reviews: reporting guideline. $B M J$ 2020;72:16890

59 Hopewell S, Loudon K, Clarke MJ. Publication bias in clinical trials due to statistical significance or direction of trial results. Cochrane Database Syst Rev 2009;1:MR000006.

60 Arbaje Al, Kansagara DL, Salanitro AH, et al. Regardless of age: incorporating principles from geriatric medicine to improve care transitions for patients with complex needs. J Gen Intern Med 2014;29:932-9. 